\title{
Análisis Psicométrico del Inventario de Orientaciones Suicidas ISO-30 en Adolescentes Escolarizados (14-18 años) y Jóvenes Universitarios Marplatenses
}

\author{
Psychometric Analysis of the Inventory of Suicide Orientation ISO-30 in Adolescent \\ Students (14-18 Years) and Young College Students from Mar del Plata
}

\author{
Aixa L. Galarza ${ }^{1}$, Mercedes Fernández-Liporace ${ }^{2}$, Claudia Castañeiras ${ }^{3}$ y Agustín Freiberg- \\ Hoffmann ${ }^{4}$
}

\section{Resumen}

El Inventario de Orientaciones Suicidas ISO-30 es una de las escalas más ampliamente utilizadas en Argentina. Este trabajo analizó sus propiedades psicométricas en una muestra de adolescentes y jóvenes universitarios de Mar del Plata, Bs As. El estudio se dividió en dos etapas. La etapa exploratoria incluyó con 821 casos $\left(62.5 \%\right.$ mujeres, $37.5 \%$ varones; $\left.M_{\text {edad }}=16.40 ; D S=1.09\right)$, y desarrolló análisis de evidencias de validez de contenido y análisis factoriales exploratorios. En la etapa confirmatoria se trabajó con 297 adolescentes y jóvenes (67\% mujeres y 33\% varones; $\left.M_{\text {edad }}=16.78 ; D S=2.02\right)$, y se realizó análisis factorial confirmatorio y de consistencia interna. Asimismo, se comparó el modelo dimensional obtenido versus el hallado en un estudio previo. Los resultados permitieron arribar a una versión reducida de la escala, ISO-19 que, si bien supone diferencias con la ISO-30, presenta evidencias psicométricas que avalan y justifican su aplicabilidad.

Palabras clave: riesgo de suicidio, adolescentes, jóvenes adultos, evaluación psicológica, psicometría

\begin{abstract}
The Inventory of Suicide Orientation ISO-30 is one of the most widely employed scales in Argentina. This paper aims to analyse the psychometric features of the scale in a sample of adolescent students and young college students from Mar del Plata, Buenos Aires. The study entailed two phases. The exploratory phase took into account 821 cases $\left(62.5 \%\right.$ female, $37.5 \%$ male; $\left.M_{\text {age }}=16.40 ; D S=1.09\right)$ and carried out evidences of content validity analyses and an exploratory factor analysis. The confirmatory phase included 297 adolescents and young adults (all students, $67 \%$ female, 33\% male; $M_{\text {age }}=16.78 ; D S=2.02$ ). A confirmatory factor analysis and internal consistency analyses were conducted. Furthermore, the dimensional model obtained was compared to the previous model. Results allowed to present a shorter version of the scale, ISO19 which, despite of its differences with the original version, ISO-30 showed adequate psychometric evidences which support and justify its applicability.
\end{abstract}

Keywords: suicide risk, adolescents, young adults, psychological assessment, psychometrics

\footnotetext{
${ }^{1}$ Lic. en Psicología. Esp. en Psicoterapia Cognitiva. Becaria Doctoral CONICET. Prof. Adjunta Facultad de Psicología, Universidad Nacional de Mar del Plata, Argentina. Bolívar 2939 5²A, Argentina. Tel.: 223-6089221. Correo: aixa_galarza@live.com.ar

${ }^{2}$ Dra. en Psicología. Investigadora Independiente CONICET. Prof. Titular Facultad de Psicología, Universidad de Buenos Aires, Argentina. Tel.: 11-30438762. Correo: mliporac@ hotmail.com

${ }^{3}$ Dra. en Psicología. Prof. Titular y Directora del Grupo de Investigación en Evaluación Psicológica GIEPsi - Instituto de Psicología Básica, Aplicada y Tecnología IPSIBAT. Facultad de Psicología, Universidad Nacional de Mar del Plata, Argentina. Tel.: 2235035098. Correo: claudia_mdq@gmail.com

${ }^{4}$ Dr. en Psicología. Investigador Asistente CONICET Facultad de Psicología, Universidad de Buenos Aires, Argentina. Tel.: 1163577196. Correo: agustinfreiberg@ gmail.com 


\section{Introducción}

En las últimas décadas se reporta un aumento progresivo de la prevalencia de los comportamientos suicidas en adolescentes y adultos jóvenes (Organización Mundial de la Salud, 2018). En Argentina, desde 2005, tales comportamientos ocupan el segundo lugar como causa de muerte en esas franjas etarias (Ministerio de Salud de la Nación, 2017). Respecto de la prevalencia de riesgo, estudios realizados en ciudades como Buenos Aires, Catamarca, Tucumán y Mar del Plata, señalaron que entre el 6 y el $15 \%$ de los adolescentes escolarizados presentaban alto riesgo de desarrollar algún comportamiento suicida (Casullo \& FernándezLiporace, 2007; Galarza, Martínez-Festorazzi, Castañeiras, \& Posada, 2014). Así, cada vez son más los países que incluyen el tópico en la agenda de salud pública como prioridad (OMS, 2018). Argentina sancionó la Ley Nacional № 27.130 de Prevención de Suicidio (Senado \& Cámara de Diputados de la Nación, 2015), que lo declara tema de interés en todo el territorio, garantiza atención biopsicosocial, investigación científica y epidemiológica, capacitación profesional para la detección y atención de personas en riesgo, y asistencia a familias víctimas de suicidios. Este escenario destaca la importancia del problema y plantea la necesidad de revisar y mejorar las estrategias de detección precoz y las intervenciones tempranas en casos de riesgo.

En cuanto a la detección de riesgo, una de las primeras dificultades reside en la operacionalización de los constructos, que afecta la construcción y adaptación de pruebas sobre suicidalidad. $\mathrm{Si}$ bien la suicidología contemporánea aún adolece de una adecuada sistematización de los conocimientos disponibles (Barzilay \& Apter, 2014; Ellis, 2008; Gvion \& Apter, 2012), paulatinamente ha logrado ciertos consensos. En este marco se concibe el suicidio como un proceso multideterminado y de máxima complejidad en el que interactúan factores cognitivos, emocionales, de personalidad, interpersonales, socioculturales, ambientales y biológicos (Ellis, 2008; Joiner, 2005; O'Connor \& Nock, 2014). En él pueden sucederse momentos de gravedad variable, que implican emociones, pensamientos y comportamientos diversos. Las nomenclaturas han abandonado la triple categorización de ideaciones suicidas, parasuicidios y suicidio (Casullo \& Scheinsohn, 2006). En cambio, la mayoría de los autores acepta la propuesta del Centers for Disease Control and Prevention (Crosby, Ortega, \& Melanson, 2011), en la que suicidio alude a un acto deliberado que realiza un individuo para poner fin a su vida; intento de suicidio supone la tentativa de autoeliminación intencionada sin resultado mortal; ideación suicida comprende la aparición de pensamientos y sentimientos vinculados con terminar con la propia existencia, y comportamientos suicidas se emplea como sinónimo de suicidalidad, abarcando todo el espectro, incluso ideaciones, planes, crisis y tentativas suicidas.

El criterio de demarcación suicida-no suicida descansa en la intencionalidad del acto. Sin embargo, varios autores discrepan con estas definiciones, que las consideran restrictivas y taxativas (e.g., Hamza, Stewart, \& Willoughby, 2012; Kapur, Cooper, O'Connor, \& Hawton, 2013). Por ejemplo, para King y Kowalchuk (1994) las ideaciones suicidas no se limitan a pensamientos sobre quitarse la vida sino que implican procesos cognitivos y estados afectivos sobre falta de sentido vital, preocupaciones sistemáticas y/o delirantes referidas a autodestrucción, muerte y elaboración de planes concretos.

Más allá de estos debates conceptuales, los especialistas acuerdan en que la aparición de ideaciones suicidas evidencia inexorablemente la activación de un proceso de suicidalidad. Si bien ello no implica el pasaje inevitable a una tentativa (Barzilay et al., 2015; Horton et al., 2016), la detección de ideaciones activas es motivo para la intervención temprana y la prevención. Por ende, los instrumentos psicométricos de screening que buscan detectar riesgo suicida evalúan la presencia de ideaciones junto con otros factores de riesgo/predictores (Goldston, 2000). Aunque Argentina cuenta con escalas de screening con propiedades psicométricas analizadas (e.g., Desuque, Vargas Rubilar, \& Lemos, 2011), solamente los inventarios MAST (Orbach et al., 1991; Casullo, Bonaldi, \& Fernández-Liporace, 2006) e ISO-30 (King \& Kowalchuk, 1994; Fernández-Liporace \& Casullo, 2006) fueron 
diseñados y posteriormente adaptados específicamente para población adolescente.

El Inventario de Orientaciones Suicidas ISO30 es el más difundido en nuestro país. Se lo utiliza en contextos clínicos, en investigación y en intervenciones comunitarias en el marco del Programa Entrenadores de Vida desarrollado por la Subsecretaría de Juventud de la Nación y la Fundación de Prevención del Suicidio. La escala original fue diseñada por King y Kowalchuk en Israel en 1994. Ella propone un modelo cognitivoconductual del suicidio en tres etapas: amenaza, crisis y resolución. La etapa de amenaza abarca la presencia de problemas no resueltos, dificultades en las estrategias de afrontamiento, aislamiento emocional, depresión y/o acting-out. La etapa de crisis alude a la experiencia de descontrol emocional, sentimientos de desesperanza y la percepción del suicidio como alternativa posible para poner fin al sufrimiento. La resolución implica la contemplación de medios y oportunidades para concretar el suicidio y la preparación para la muerte. Desde este enfoque, el ISO-30 intenta evaluar la orientación suicida en adolescentes de 13 a 18 años a partir de cinco dimensiones integradas por 6 ítems cada una: Desesperanza, Baja Autoestima, Incapacidad para Afrontar Problemas Emocionales, Soledad y Aislamiento Social e Ideación Suicida. Esta multidimensionalidad constituye una fortaleza de la escala, en oposición a otras que contemplan una o dos dimensiones que no logran abarcar la complejidad del fenómeno (e.g. Goldston, 2000; Osman et al., 2005). Pese a tal ventaja, existen pocos estudios sobre sus propiedades psicométricas en muestras clínicas. En esta línea, Osman et al. (2005) analizaron evidencias de validez externa, interna -grupo clínico vs controles; estudios de validez convergente y predictiva-, así como análisis discriminante y consistencia interna.

La adaptación local de la escala fue desarrollada por Casullo (1997). Casi una década más tarde se analizó, en una muestra de 642 adolescentes de Buenos Aires entre 13 y 20 años, la capacidad discriminativa de los reactivos, la estructura factorial y la consistencia interna de dimensiones y escala total (Fernández-Liporace \& Casullo, 2006). En el análisis de homogeneidad, 6 de 29 reactivos mostraron un $r h_{i} \leq .30$ (ítems 1,3 ,
7, 16, 23, 28). El análisis de la dimensionalidad incluyó análisis de Componentes Principales y rotación Varimax, obteniéndose una estructura pentafactorial que explicaba el $45.02 \%$ de la varianza, y resultaba parcialmente consistente con el modelo propuesto, ya que el agrupamiento de ítems no coincidía con el de la versión original (F1, Ideación Suicida $\alpha=.88$; F2, Desesperanza $\alpha=74$; F3, Esperanza $\alpha=.67$; F4, Pertenencia Interpersonal, $\alpha=.43 ; \quad$ F5, Capacidad de Autocontrol y Relación con los Otros, $\alpha=50$ ).

A pesar de las sugerencias preliminares de modificación de ítems $(10,18,28,29) \mathrm{y}$ de denominación de las escalas propuestas por Fernández-Liporace y Casullo (2006), el instrumento continúa utilizándose en su versión original de 30 elementos. Incluso la revisión de literatura pone de manifiesto que la adaptación argentina de 1997 es utilizada asiduamente en países de la región como Ecuador (Peña-Castro, Cordero-Tapia, \& Tapia-Cuzco, 2009), Perú (Huanca-Morales, 2017) y Colombia (PaniaguaSuárez, González-Posada, \& Rueda-Ramírez, 2014). Solamente se ha informado un estudio psicométrico realizado en población colombiana, que ofrece evidencias de validez de constructo, de criterio y resultados en cuanto a confiabilidad en cuanto a consistencia interna y estabilidad (Paniagua-Suárez, González-Posada, \& RuedaRamírez, 2016). Por lo tanto, dada la antigüedad y el carácter exploratorio de esos estudios, la ISO30 debiera someterse a nuevos análisis. El envejecimiento e insuficiencia de los trabajos previos sumados a la prioridad brindada a los comportamientos suicidas y al suicidio adolescente como problemas de salud pública plantean la urgencia de revisar, actualizar y mejorar los instrumentos de evaluación disponibles para justificar su aplicación en población local. Por ello este trabajo persigue tres objetivos: 1) analizar evidencias de validez de contenido a través de un juicio experto; 2) examinar evidencias de validez de constructo y consistencia interna mediante un análisis factorial exploratorio; y 3) contrastar y analizar el ajuste del modelo pentafactorial extraído en la adaptación de Fernández-Liporace y Casullo versus el modelo obtenido en el presente trabajo; analizar, además, la consistencia interna de los factores del modelo final. 


\section{Método}

\section{Tipo de estudio y diseño}

Instrumental (Montero \& León, 2007), de tipo psicométrico, siguiendo las recomendaciones de Martínez Arias (2014), Cohen y Swerdlik (2006) y Muñiz, Elousa, \& Hambleton (2013). Diseño no experimental, correlacional y explicativo (Hair, Anderson, Tatham, \& Black, 1999).

\section{Participantes}

Para la etapa exploratoria se trabajó con 821 casos $\quad(62.5 \%$ mujeres, $37.5 \%$ varones; $M_{\text {edad }}=16.40 ; \quad D S=1.09 ; \quad M d n=16 ; \quad$ Mín=15; Máx=21). El $63.5 \%$ asistía a instituciones educativas de gestión privada y el $36.5 \%$, a instituciones educativas de gestión pública, en ambos casos de nivel medio.

Para la etapa confirmatoria se trabajó con 297 adolescentes de 14 a 18 años y jóvenes universitarios $(67 \%$ mujeres y $33 \%$ varones; $M_{\text {edad }}=16.78 ; \quad D S=2.02 ; \quad M d n=17 ; \quad$ Mín=14; Máx=21). El $50.16 \%$ asistía a instituciones educativas secundarias de gestión pública, el $17.86 \%$, de gestión privada; el $31.98 \%$ correspondía a ingresantes y estudiantes de primer cuatrimestre de primer año de la Facultad de Psicología de la UNMDP, que hubieran terminado la educación secundaria el año anterior.

Se seleccionó dicho rango etario debido a que, de acuerdo con los datos oficiales del Ministerio de Salud de la Nación (2017) y de la OMS (2018), es el grupo poblacional de mayor riesgo en función de las tasas de prevalencia de muertes por suicidio.

\section{Instrumentos}

Se administró un protocolo de evaluación que incluía un registro de datos básicos para indagar información sociodescriptiva familiar, situaciones vitales e involucramiento en comportamientos autodestructivos, con el fin de contar con un criterio de riesgo, externo al instrumento.

Inventario de Orientaciones Suicidas ISO 30 (King \& Kowalchuk, 1994; adaptación Casullo, 1997; Fernández-Liporace \& Casullo, 2006). Como se describió anteriormente, evalúa la presencia de factores de riesgo vinculados a ideaciones y comportamientos suicidas. Para este estudio, se usa como punto de partida la adaptación de Casullo (1997). Consta de 30 ítems agrupados equitativamente en cinco dimensiones, que son los que, pese a los estudios locales, se emplean en la práctica: Baja Autoestima $(1,6,11$, 16, 21 y 26), Desesperanza (ítems 2, 7, 12, 17, 22 y 27), Incapacidad para Afrontar Problemas Emocionales (ítems 3, 8, 13, 18, 23 y 28), Soledad y Aislamiento Social (ítems 4, 9, 14, 19, 24 y 29), e Ideaciones Suicidas (subescala de ítems críticos: $5,10,15,20,25$ y 30). La escala likert empleada admite cuatro opciones de respuesta en función del grado de acuerdo con cada afirmación (entre 0 y 3$)$.

\section{Procedimiento}

La aplicación se realizó en forma colectiva en los tres últimos años de educación secundaria de 15 instituciones educativas de la ciudad de Mar del Plata y en el primer año de la carrera de Psicología de la Universidad Nacional de Mar del Plata. Se organizó por cursos en un único encuentro de aproximadamente una hora.

En todos los casos la participación fue voluntaria, sin retribución económica y bajo consentimiento informado, tanto de los adolescentes como de padres o tutores, en los casos en que ello correspondiera. Todas las etapas del estudio siguieron las recomendaciones para la investigación con seres humanos establecidas en la Declaración de Helsinki (WMA, 2014), los Códigos de Ética de la APA (2010) y de Fe.P.R.A (2013). Se garantizó el anonimato y la confidencialidad de los datos en función de la Ley Nacional 25.326 de Protección de Datos Personales.

\section{Análisis de datos}

Para el análisis de validez de contenido propuesto en el objetivo 1, se sometieron los reactivos a un proceso de valoración por cinco jueces expertos, tres del campo de la psicometría y dos provenientes de la suicidología. Se elaboró una planilla en la que se presentaron las dimensiones que componen la ISO-30, su finalidad y población-objeto, y el listado de ítems. Se solicitó a los jueces que valoraran cada afirmación según la relevancia o representatividad en relación con el constructo teórico y la dimensión que pretendía medir, la redacción y 
adecuación terminológica para población adolescente, y la dimensión a la que cada una pertenecería. Luego, se calculó el coeficiente $V$ de Aiken para estimar el acuerdo interjueces. Respecto del objetivo 2, primero se realizó el análisis paralelo para determinar el número de factores a extraer y se decidió ejecutar un análisis factorial exploratorio con método de extracción Minimum Rank Factor Analysis robusto para variables ordinales, con rotación Promax y normalización Kaiser. Además se eliminaron todos aquellos reactivos cuya saturación fuera inferior a .30 o bien que superara dicho guarismo en dos factores de modo simultáneo (Yong \& Pearce, 2013). Por último, para responder al objetivo 3 de comparación de modelos rivales (modelo pentafactorial original propuesto por Casullo vs modelo obtenido), se efectuó un análisis factorial confirmatorio mediante el método de estimación de Máxima Verosimilitud Robusto (Hoyle, 2012; Li, 2016). Debido a que los ítems presentaban un nivel de medición ordinal se aplicó, para los últimos dos objetivos, una metodología robusta basada en el cálculo de correlaciones policóricas (Holgado-Tello, Chacón-Moscoso, Barbero-García, \& Vila-Abad, 2010). Para analizar la consistencia interna, se calcularon los coeficientes alfas ordinales a través de un procedimiento de modelización que posibilita obtener estimaciones a partir de la varianza explicada por cada reactivo con independencia de la varianza de error (Herrero, 2010).

\section{Resultados}

\section{Objetivo 1. Validez de contenido}

En primer lugar, se calculó el coeficiente $V$ de Aiken para cada ítem con el fin de estimar el grado de acuerdo entre los evaluadores. El criterio para conservar los reactivos se estableció en un coeficiente $V \geq .80$, que implica el acuerdo mayoritario por parte de los jueces en cuanto a la representatividad y la claridad de la pertenencia de un ítem en relación con la dimensión de pertenencia. Y se adoptó el valor de $V \leq .60$ para eliminar elementos. A partir de este estudio solamente el ítem 28 (Nunca sentí que estaba a punto de quebrarme, de hacerme pedazos) surgió como problemático debido a su contenido y redacción, por lo que fue eliminado.

\section{Objetivo 2. Evidencias de validez de constructo: Análisis factorial exploratorio}

Previo al análisis factorial exploratorio se realizó un análisis paralelo (Tabla 1) para analizar el número de factores cuya extracción resultara óptima desde el punto de vista estadístico (Horn, 1965). Se aplicó una metodología basada en la simulación de valores a partir de una distribución teórica conocida. Para ello se generaron 500 matrices de correlaciones aleatorias que serían comparadas con los datos reales (Baglin, 2014). La determinación de factores se realizó tomando como referencia el percentil 95 de los autovalores aleatorizados dado que arroja una medida conservadora de la media y evita que la interpretación de los resultados se vea afectada por el error de Tipo I (Green, Thompson, Levy, \& Lo, 2015). Como consecuencia de lo anterior, el resultado del análisis paralelo indicó la conveniencia de extraer cuatro factores.

Tabla 1. Análisis paralelo

\begin{tabular}{cccc}
\hline & $\begin{array}{c}\text { Autovalores } \\
\text { reales }\end{array}$ & $\begin{array}{c}\text { Autovalores } \\
\text { medios } \\
\text { aleatorios }\end{array}$ & $\begin{array}{c}\text { P95 de } \\
\text { autovalores } \\
\text { aleatorios }\end{array}$ \\
\hline 1 & 6.98 & 1.28 & 1.32 \\
2 & 1.74 & 1.23 & 1.27 \\
3 & 1.46 & 1.19 & 1.22 \\
4 & 1.23 & 1.16 & 1.18 \\
\hline
\end{tabular}

Tomando en cuenta el resultado anterior se forzó en el análisis factorial exploratorio la extracción a cuatro factores. Se aplicó el método de extracción Minimum Rank Factor Analysis robusto para variables ordinales, con rotación Promax y normalización Kaiser, y se observó un buen ajuste entre el número de ítems en análisis y el tamaño muestral $(\mathrm{KMO}=.883$; Test de Esfericidad de Bartlett $\left.X^{2}=3867.6 ; g l=190 ; p<.01\right)$.

La solución extraída conservó 19 elementos y explicó el 76.8\% de la varianza común (el Factor 1 explicó 22.9\%; el Factor 2, el 19.2\%; Factor 3, $18.2 \%$ y el Factor 4, 16.5\%) (Tabla 2). Fueron eliminados los ítems 3, 6, 9, 10, 13, 16, 17, 19, 21, 23 y 28 por no cumplir sus cargas factoriales en función de los criterios establecidos para su conservación. A partir del modo en que los elementos se agruparon se decidió etiquetar el Factor 
Tabla 2. Solución de 4 factores

\begin{tabular}{|c|c|c|c|c|c|}
\hline \multirow{2}{*}{\multicolumn{2}{|c|}{$\begin{array}{l}\text { Ítems } \\
\text { ISO-30 }\end{array}$}} & \multirow{2}{*}{ F1 } & \multirow{2}{*}{$\begin{array}{c}\text { F2 } \\
\text { SYA } \\
\end{array}$} & \multirow{2}{*}{$\mathrm{F} 3$} & \multirow{2}{*}{ F4 } \\
\hline & & & & & \\
\hline 15 & Para impedir que las cosas empeoren creo que el suicidio es la solución & .84 & & & \\
\hline 30 & Si mis cosas empeoran creo que me mataría & .78 & & & \\
\hline 25 & Para no sentirse mal o solo/a la solución es morirse & .74 & & & \\
\hline 20 & Pienso en morirme como una forma de resolver mis problemas & .65 & & & \\
\hline 4 & Confió en que los que se preocupan por mí no me van a abandonar si tengo un fracaso & & .64 & & \\
\hline 14 & Las personas que son importantes para mi comprenden mis sentimientos & & .63 & & \\
\hline 26 & Nadie me querría si realmente me conociera & & .57 & & \\
\hline 29 & $\begin{array}{l}\text { Los que me quieren se equivocan. Con el tiempo se van a dar cuenta de cómo soy } \\
\text { realmente }\end{array}$ & & .53 & & \\
\hline 5 & Las personas con las que me relaciono no me necesitan en absoluto & & .51 & & \\
\hline 11 & Tengo las cualidades necesarias para ser feliz & & & 67 & \\
\hline 7 & Mi vida se ha desarrollado en general en la forma que yo quise & & & 63 & \\
\hline 27 & Posiblemente llegue a ser la clase de persona que quiero ser & & & .58 & \\
\hline 2 & En el futuro hay para mí muchas posibilidades de ser feliz & & & .58 & \\
\hline 22 & Siento que puedo controlar mi vida & & & .40 & \\
\hline 18 & Cuando mi vida enfrenta alguna dificultad me confundo & & & & .60 \\
\hline 12 & $\begin{array}{l}\text { Cuando algo malo me sucede siento que mis esperanzas de una vida mejor son poco } \\
\text { reales }\end{array}$ & & & & .59 \\
\hline 8 & Quisiera poder hacer que duren más los buenos momentos, pero no puedo & & & & .54 \\
\hline 24 & Cuando algo me sale mal quiero esconderme, desaparecer & & & & .53 \\
\hline 1 & Debo ser un/a soñador/a, dado que siempre espero cosas que no ocurren & & & & .51 \\
\hline
\end{tabular}

Nota. IS=Ideaciones Suicidas; SYA=Soledad y Aislamiento Social; D=Desesperanza; BA=Baja Autoeficacia.

Tabla 3. Índices de ajuste de los modelos tetra y pentafactorial

\begin{tabular}{lcccccccc}
\hline \multirow{2}{*}{ Modelo } & \multicolumn{8}{c}{ Índices de Ajuste } \\
\cline { 2 - 10 } & $\chi^{2}$ & $g l$ & NFI & NNFI & CFI & IFI & MFI & RMSEA [IC] \\
\hline 5 Factores & $2465 * *$ & 171 & .91 & .97 & .97 & .97 & .90 & $.03[.02-.03]$ \\
4 Factores & $2465.03^{* *}$ & 171 & .93 & .99 & .99 & .99 & .97 & $.02[.00-.03]$ \\
\hline
\end{tabular}

$* * p<0.01$.

1 como Ideaciones Suicidas, el Factor 2 como Soledad y Aislamiento Social, el 3 como Desesperanza y el 4 como Baja Autoeficacia.

\section{Objetivo 3. Análisis factorial confirmatorio. Comparación de modelos}

Con el fin de evaluar el ajuste de los datos empíricos al modelo tetrafactorial derivado del procedimiento exploratorio, y comparar dicho ajuste con el modelo pentafactorial extraído en la adaptación previa (Fernández-Liporace \& Casullo, 2006), se efectuó un análisis factorial confirmatorio. Para ello se empleó el método de estimación de Máxima Verosimilitud Robusto por ser recomendable cuando las variables ordinales son analizadas a partir de correlaciones policóricas con un tamaño muestral apropiado ( $n \geq 200$ ) (Hoyle, 2012; Li, 2016).

$\mathrm{El}$ ajuste de los modelos fue evaluado a través de los índices NFI (Normed Fit Index), NNFI (Non-Normed Fit Index), CFI (Comparative Fit
Index), IFI (Bollen's Fit Index), MFI (McDonald's Fit Index) y RMSEA (Root MeanSquare Error of Aproximation). Se estimaron estos índices para los modelos pentafactorial (Figura 1) propuesto por Fernández-Liporace \& Casullo (2006), y tetrafactorial (Figura 2) derivado del análisis factorial exploratorio antes presentado.

Como resultado de los análisis se observa primeramente que los índices de ajuste $N F I$, NNFI, CFI, IFI y MFI superan en ambos modelos el valor de .90 , guarismo que distingue un buen ajuste de uno mediocre (Brown, 2015), aunque pueden apreciarse valores más elevados para el modelo tetrafactorial. Por otra parte el estadístico RMSEA para ambos fue inferior a .05 , valor máximo admisible para considerar un buen ajuste del modelo a los datos empíricos (Kline, 2011) (Tabla 3). En cuanto al análisis de parsimonia de los modelos, se estimaron los índices CAIC Independiente y CAIC Modelo. Mayor distancia 


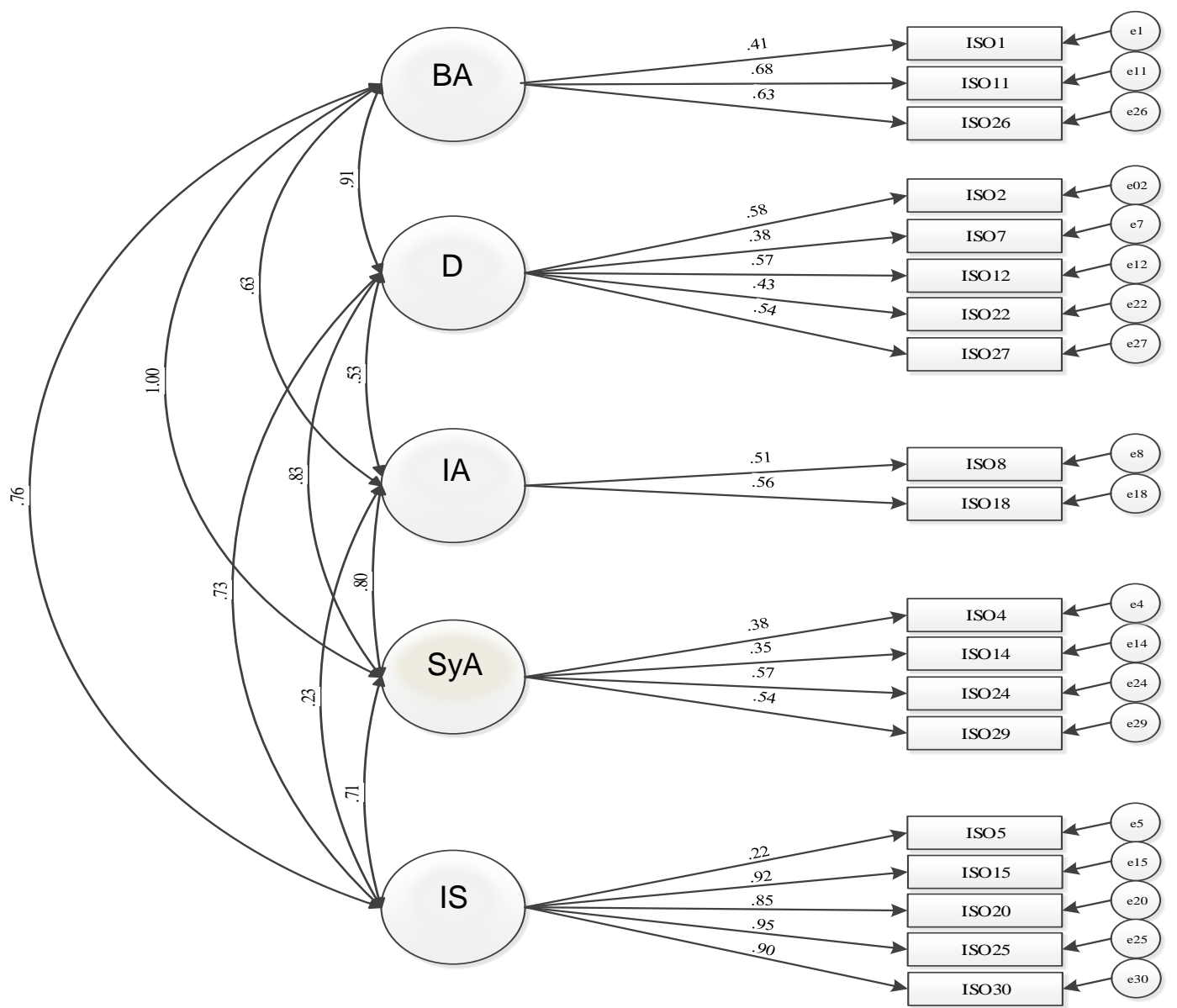

Figura 1. Modelo pentafactorial (Fernández Liporace \& Casullo, 2006).

BA=Baja Autoestima; D=Desesperanza; IAP=Incapacidad para afrontar los problemas; SYA=Soledad y Aislamiento Social; IS=Ideaciones Suicidas

Tabla 4. Índices de parsimonia de los modelos tetra y pentafactorial.

\begin{tabular}{lcc}
\hline \multirow{2}{*}{ Modelo } & \multicolumn{2}{c}{ Índices de Parsimonia } \\
\cline { 2 - 3 } & CAIC Modelo & CAIC Independiente \\
\hline 5 Factores & -750.503 & 1320.407 \\
4 Factores & -813.683 & 1320.407 \\
\hline
\end{tabular}

entre ambos índices indica una mayor parsimonia del modelo estimado. En este sentido, el modelo tetrafactorial mostró una mayor diferencia y por ende, una mayor parsimonia que el pentafactorial (Tabla 4) (Hooper, Coughlan, \& Mullen, 2008).

Con respecto al examen de los parámetros estimados para cada modelo, se destaca que, en ambos casos, la totalidad de las variables observadas realiza un aporte significativo $(p<.05)$ a la explicación de las variables latentes. Solamente se registra para el modelo pentafactorial una ecuación infractora -parámetro igual a 1- entre las variables latentes $\mathrm{F} 1$ y $\mathrm{F} 4$ (Brown, 2015), que no ha sido removida ni ha recibido tratamiento estadístico especial, ya que se
Tabla 5. Coeficientes de determinación

\begin{tabular}{llcc}
\hline $\begin{array}{l}\text { Modelo } \\
\text { Tetrafactorial }\end{array}$ & \multicolumn{3}{c}{$\begin{array}{l}\text { Modelo } \\
\text { Pentafactorial }\end{array}$} \\
\hline Ítems & $R^{2}$ & Ítems & $R^{2}$ \\
\hline ISO1-F2 & .22 & ISO1-F1 & .16 \\
ISO2-F3 & .37 & ISO2-F2 & .33 \\
ISO4-F4 & .18 & ISO4-F4 & .14 \\
ISO5-F4 & .21 & ISO5-F5 & .04 \\
ISO7-F3 & .18 & ISO7-F2 & .14 \\
ISO8-F2 & .19 & ISO8-F3 & .25 \\
ISO11-F3 & .61 & ISO11-F1 & .46 \\
ISO12-F2 & .50 & ISO12-F2 & .32 \\
ISO14-F4 & .13 & ISO14-F4 & .12 \\
ISO15-F1 & .84 & ISO15-F5 & .84 \\
ISO18-F2 & .21 & ISO18-F3 & .31 \\
ISO20-F1 & .71 & ISO20-F5 & .71 \\
ISO22-F3 & .21 & ISO22-F2 & .18 \\
ISO24-F2 & .51 & ISO24-F4 & .32 \\
ISO25-F1 & .91 & ISO25-F5 & .90 \\
ISO26-F4 & .54 & ISO26-F1 & .39 \\
ISO27-F3 & .38 & ISO27-F2 & .29 \\
ISO29-F4 & .43 & ISO29-F4 & .28 \\
ISO30-F1 & .80 & ISO30-F5 & .80 \\
\hline
\end{tabular}




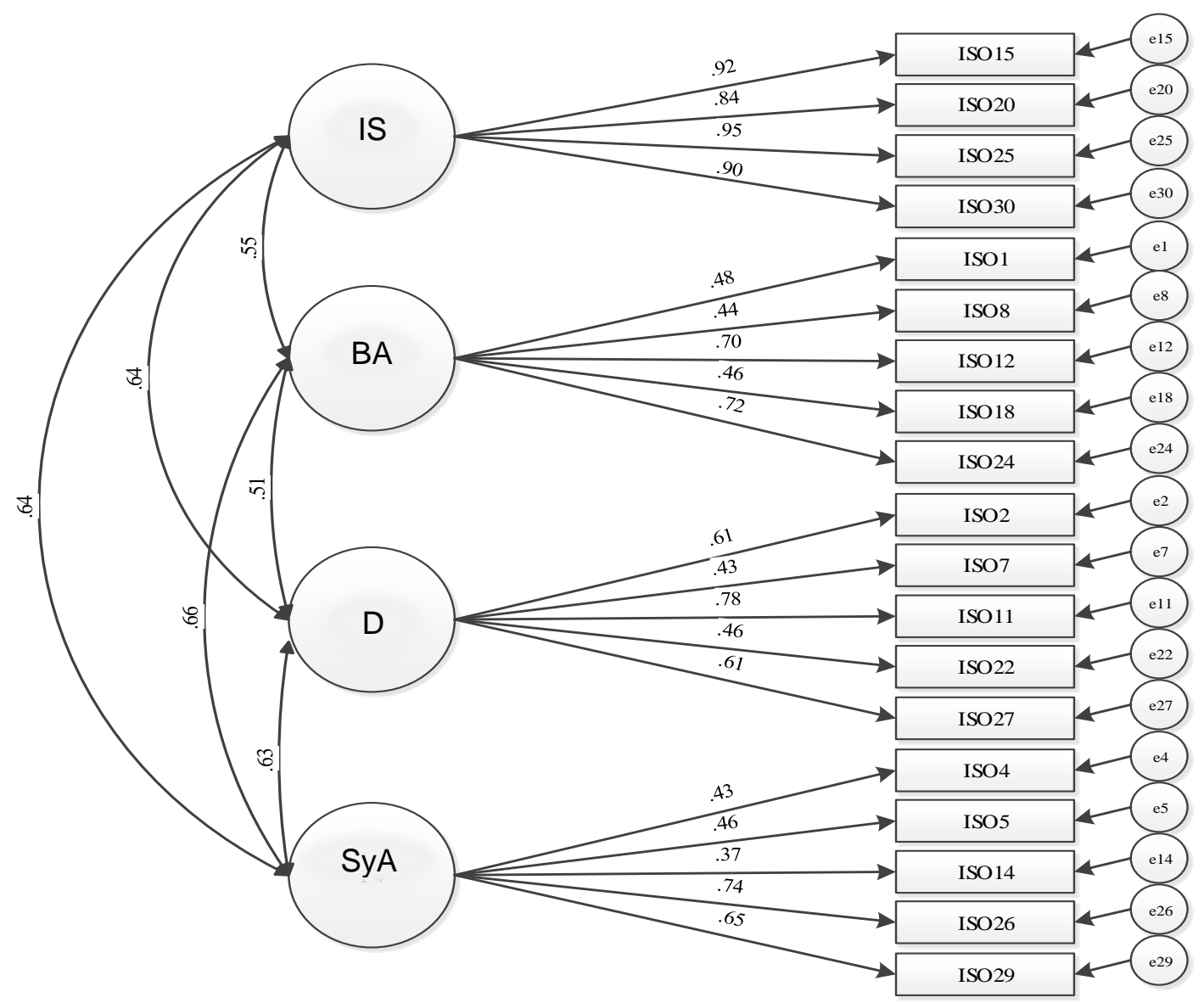

Figura 2. Modelo tetrafactorial extraído del análisis factorial exploratorio.

IS=Ideaciones Suicidas; BA=Baja Autoeficacia; D=Desesperanza; SYA=Soledad y Aislamiento Social.

ha demostrado que su ocurrencia no afecta significativamente el ajuste del modelo (Chen, Bollen, Paxton, Curran, \& Kirby, 2001). Al analizar los coeficientes de determinación $\left(R^{2}\right)$ de cada parámetro estimado, se advierte que el modelo tetrafactorial supera al pentafactorial en el aporte que 14 variables observadas realizan a la explicación de las latentes, mientras que el modelo pentafactorial supera al tetrafactorial en otras dos variables observadas. Por otra parte, 3 variables observadas realizan un similar aporte a la explicación de las variables latentes en ambos modelos.

$\mathrm{Si}$ se considera que el aporte que debieran realizar las variables observables a la explicación de las latentes se vuelve adecuado si llega al 50\% de su variabilidad $\left(R^{2}=.50\right)$ (Hair, Hut, Ringle, \& Sarstedt, 2016; Hancock \& Mueller, 2013), es posible apreciar que en el modelo tetrafactorial alcanzan tal valor 7 variables mientras que en el pentafactorial solamente lo logran cuatro (Tabla 5).

En relación con las covarianzas de los modelos, resulta interesante mencionar que su análisis posibilita indagar evidencias de validez convergente y discriminante en pos de detectar la necesidad de fundir dos variables latentes en una, o bien evaluar la posibilidad de insertar variables intermedias. Para esto se tomaron a las covarianzas iguales o superiores a .85 como indicadores de validez convergente, $\mathrm{y}$ a las iguales o inferiores a .50 de validez discriminante, en tanto que las covarianzas con valores intermedios se consideraron indicadores de independencia entre variables latentes (Abalo-Piñeiro, LéviMangin, Rial-Boubeta, \& Varela-Mallou, 2006). Para el modelo tetrafactorial todas las covarianzas se ubicaron en los guarismos establecidos para garantizar la independencia entre variables. Con respecto al modelo pentafactorial, se encontró evidencia de validez convergente entre F1 y F2 (.91) y discriminante entre F3 y F5 (.23). Como ya se ha dicho la covarianza entre F1 y F4 (1.00) no puede interpretarse ya que corresponde a una ecuación infractora (Brown, 2015).

Finalmente, se calcularon para ambos modelos los coeficientes alfas ordinales 
correspondientes a cada dimensión. Así, para el modelo tetrafactorial se obtuvieron coeficientes de .95 para el Factor Ideaciones Suicidas, .65 para el Factor Soledad y Aislamiento Social, .71 para el Factor Desesperanza y .69 para Baja Autoeficacia. Por su parte el modelo pentafactorial arrojó índices de .58 para el Factor 1, .62 para el Factor 2, .44 para el Factor 3, .51 para el Factor 4 y .87 para el Factor 5.

\section{Discusión}

El trabajo que se presenta se propuso retomar la línea de investigación sobre las propiedades psicométricas del Inventario de Orientaciones Suicidas ISO-30 para adolescentes, que iniciara Casullo en 1997 y continuara brevemente con el estudio psicométrico preliminar de FernándezLiporace y Casullo en 2006.

Primero se llevó acabo el juicio experto para estimar evidencias de validez de contenido, lo que condujo a la eliminación de un reactivo de la dimensión Incapacidad para Afrontar los Problemas Emocionales.

Luego, se procedió al estudio de validez de constructo. Tras analizar diferentes matrices, la estructura factorial que emergió como más adecuada, a diferencia del estudio de FernándezLiporace y Casullo (2006), quedó compuesta por cuatro factores. El primero agrupó 4 ítems específicos de la dimensión Ideaciones Suicidas $(15,20,25$ y 30$)$. A diferencia de la versión adaptada por Casullo (1997) y FernándezLiporace y Casullo (2006), solamente se mantuvieron los reactivos que indagan Ideaciones Suicidas en sentido restrictivo, descartando aquellos que versan sobre la muerte en general. El segundo factor es claramente identificado como sentimientos de soledad y bajo sentido de pertenencia social, y quedó compuesto por la mitad de los ítems originales de la dimensión de Soledad y Aislamiento Social (4, 14 y 29), uno de Baja Autoestima (26) y uno de Ideaciones Suicidas (5). La inclusión de estos dos ítems en este factor resulta altamente congruente dado que ambos remiten a la percepción de lo que Joiner (2005), autor de una de las teorías suicidológicas más relevantes en la actualidad, denomina burdensomeness y thwarted belongingness, entendidos como ser una carga para los demás y la falta de pertenencia social, respectivamente. El tercer factor agrupó cinco reactivos que refieren a una visión positiva u optimista de sí mismo y del futuro, cuatro de los cuales corresponden a la dimensión original Desesperanza del modelo pentafactorial (Casullo, 1997; FernándezLiporace, 2006), por lo que se conserva su nombre original. Por último, el cuarto factor es el más ecléctico, al integrarse con elementos provenientes de distintas dimensiones. Sin embargo, al analizar su contenido claramente se puede observar que todos aluden a una evaluación negativa de los propios recursos, bajo sentido de autoeficacia y falta de dominio de sí mismo ante la percepción de situaciones problemáticas, con lo cual se denomina Baja Autoeficacia.

Una vez depurado el instrumento en la instancia exploratoria, se aplicó la versión resultante a una nueva muestra a fin de testear el ajuste de los datos empíricos al modelo tetrafactorial. Para esto se efectuó el análisis factorial confirmatorio y se comparó el modelo derivado del estudio exploratorio con el pentafactorial propuesto por Fernández-Liporace y Casullo (2006). De la comparación de ambos modelos se obtuvo primeramente un mayor nivel de ajuste y parsimonia del tetrafactorial por sobre el pentafactorial. Además, al examinar los parámetros estimados se registró que, si bien para ambos modelos todos ellos resultaron significativos, en el tetrafactorial los valores fueron más altos siendo ello indicador de una mejor representatividad de las variables observadas respecto de las latentes, aportándoles un mayor porcentaje de su varianza individual a su explicación. Del examen de las covarianzas se puede apreciar también que las dimensiones del modelo tetrafactorial son independientes las unas de las otras, lo que no sucede en el caso del modelo pentafactorial donde debe evaluarse la posibilidad de impactar o fundir el Factor 1 con el 2 , y de incluir una variable latente intermedia entre los Factores 3 y 5.

En cuanto al análisis de la consistencia interna de los factores hallados en el análisis factorial confirmatorio, se calcularon los coeficientes alfas ordinales a través de un procedimiento de modelización permitiendo obtener estimaciones a partir de la varianza explicada por cada ítem con 
independencia de la varianza de error (Herrero, 2010). De este modo, se obtuvieron coeficientes de .95 para el Factor Ideaciones Suicidas, .65 para el Factor Soledad y Aislamiento Social, .71 para el Factor Desesperanza y .69 para Baja Autoeficacia. Los índices alfas ordinales informados para cada dimensión pueden ser considerados apropiados a la luz del bajo número de ítems que integra cada una de ellas (Coulacoglou \& Saklofske, 2017).

Como puede apreciarse, el modelo tetrafactorial extraído del procedimiento exploratorio presenta un mejor ajuste a los datos empíricos y mayor parsimonia que el pentafactorial hallado en la investigación de Fernández-Liporace y Casullo (2006). Sin embargo, es importante destacar que a pesar de las modificaciones a nivel de los elementos, el modelo alternativo de 4 factores guarda similitudes a nivel teórico-conceptual con la versión adaptada de Casullo (1997) y las informadas por Fernández-Liporace y Casullo (2006) para población argentina, por Osman et al. (2005) para población norteamericana y por Paniagua-Suárez et al. (2016) por para población colombiana. A su vez, la estructura resultante de dimensiones y reactivos es consistente con los desarrollos teóricos actuales sobre el tema. Autores representativos acuerdan en la importancia crucial de evaluar aspectos vinculados a la suicidalidad como desesperanza, apoyo social, aislamiento, autoeficacia y capacidad percibida para resolver problemas (e.g., Horton et al., 2015; Joiner, 2005; O'Connor, Fraser, White, MacHale, \& Masterton, 2008; O'Connor \& Nock, 2014; Opperman, Czyz, Gipson, \& King, 2015), para lograr una estimación más precisa del grado de riesgo suicida de los adolescentes y jóvenes que contemple la multidimensionalidad y complejidad de la problemática.

El presente estudio cuenta con una serie de limitaciones. Es menester la inclusión de una muestra con intento de suicidio previo como criterio externo. En primer lugar, contar con un grupo de tentativa previa posibilitaría el análisis más profundo de la representatividad del ítem respecto de la dimensión evaluada, así como de su capacidad discriminativa. En segundo término, una muestra de mayor tamaño y más heterogénea en cuanto a edades, región, sin escolaridad y condición clínica (con intento de suicidio previo vs sin intento previo) hubiera permitido efectuar análisis de invarianza factorial que posibilitaría conocer si el modelo tetrafactorial es susceptible de generalizarse a partir de su replicabilidad en otros grupos de sujetos, garantizando así su invariabilidad y fiabilidad al momento de medir el constructo en esos grupos (Abalo-Piñeiro et al., 2006). En tercer lugar, tener como criterio externo una muestra de adolescentes con intento previo haría posible trabajar con grupos contrastados y analizar con rigurosidad evidencias de validez externa. A la vez, ello permitiría el establecimiento de puntajes de corte basados en la sensibilidad y especificidad de la escala para clasificar satisfactoriamente los casos en un gradiente de riesgo. Por otro lado, sería deseable realizar un estudio de DIF, para determinar si existen ítems con diferente funcionamiento por género. Por último, si bien se presentan resultados sobre evidencias de validez convergente $\mathrm{y}$ discriminante al interior del instrumento, no se efectuado dicho análisis adoptando criterios externos dado que excedía el propósito del presente estudio. Futuras investigaciones se encargaran de sumar evidencias de validez convergente y discriminante mediante otros instrumentos que evalúen constructos teóricamente relacionados.

El Inventario de Orientaciones Suicidas ISO30 es una de las escalas más utilizadas en nuestro país para estimar el nivel de riesgo suicida en adolescentes. Los resultados hallados permiten sostener que la versión reducida que surge de este estudio psicométrico presenta evidencias de validez de contenido, de validez de constructo convergente y divergente- y consistencia interna que avalan y justifican su aplicabilidad. Si bien es imperativa la realización de estudios de validez externa, es posible sostener que se trata de una herramienta útil, breve y sencilla para evaluar la multidimensionalidad del potencial suicida en adolescentes y jóvenes de nuestro medio de 14 a 21 años, quienes se encuentran dentro del rango de mayor riesgo según los datos oficiales (Ministerio de Salud de la Nación, 2017; OMS, 2018). No solamente brinda una evaluación de la presencia de ideaciones suicidas activas y su intensidad, sino que además informa sobre 
diferentes factores asociados, como la percepción de dominio y autoeficacia, el nivel de desesperanza y la presencia de sentimientos de soledad y aislamiento. Contar con esta información hace posible interpretar diferentes perfiles de riesgo y orientar las intervenciones con más precisión. A su vez, se potencia la capacidad de transferencia de la escala a los ámbitos clínico, educacional y de investigación.

De esta manera, el presente estudio constituye un punto de inicio sólido para futuras líneas de investigación, que resultan imperativas y urgentes. Particularmente, como se mencionó, es fundamental realizar estudios de grupos contratados que incluyan un grupo de no riesgo, un grupo de adolescentes y jóvenes con ideaciones suicidas sin intento previo, y un grupo con intento previo. Ello posibilitaría el análisis riguroso de la validez de constructo y de criterio concurrente y predictiva - de la escala.

\section{Referencias}

Abalo-Piñeiro, J., Lévi-Mangin, J. P., RialBoubeta, A., \& Varela-Mallou, J. (2006). Invarianza factorial con muestras múltiples. En J. P. Lévy Mangin y J. Varela Mallou (Eds.), Modelización con estructuras de covacianzas en ciencias sociales (pp. 259278). Coruña: Netbiblo.

American Psychological Association. (2010). Ethical Principles of Psychologists and Code of Conduct. Recuperado el día 04 de abril de 2011 de http://www.apa.org/ethics/code/index.aspx

Asociación Médica Mundial-WSA. (2014). Declaración de Helsinki. Recuperado el día 07 de Octubre de 2015 de http://www.wma.net/es/30publications/10poli cies/b3/

Baglin, J. (2014). Improving your exploratory factor analysis for ordinal data: A demostration using FACTOR. Practical Assessment, Research \& Evaluation, 19(5). Recuperado de http://pareonline.net/pdf/v19n5.pdf

Barzilay, S., \& Apter, A. (2014). Psychological models of suicide. Archives of Suicide Research, 18(4), 295-312. doi:10.1080/13811118.2013.824825

Barzilay, S., Feldman, D., Snir, A., Apter, A., Carli, V., Hoven, C. W., Wasserman, C., Sarchiapone, M., \& Wasserman, D. (2015). The interpersonal theory of suicide and adolescent suicidal behavior. Journal of Affective Disorders186, 68-74. doi:10.1016/j.jad.2015.04.047

Brown, T. (2015). Confirmatory factor analysis for applied research. New York: Guilford Press.

Casullo, M. M. (1997). Riesgo suicida en estudiantes universitarios. Epidemiología y prevención. Desarrollos en Psiquiatría Argentina, 2(4), 33-38.

Casullo, M. M., Bonaldi, P., \& FernándezLiporace, M. (2006). Comportamientos suicidas en la adolescencia. Buenos Aires: Lugar Editorial.

Casullo, M. M., \& Fernández-Liporace, M. (2007). Investigación sobre riesgo suicida en adolescentes. Prácticas psicológicas en la escuela. Investigaciones en Psicología, 2 (2), 33-41.

Casullo, M. M., \& Scheinsohn, M. J. (2006). Comportamientos suicidas en situaciones de internación psiquiátricas. Revista Iberoamericana de Diagnóstico y Evaluación - e Avaliação Psicológica, 22(2), 29-48.

Chen, F., Bollen, K., Paxton, P., Curran, P., \& Kirby, J. (2001). Improper solution in structural equation models. Sociological Methods \& Research, 29(4), 468-508.

Cohen, R. J., \& Swerdlik, M. E. (2006). Pruebas y evaluación psicológicas. México: MacGraw Hill Interamericana.

Costello, A., \& Osborne, J. W. (2005). Best practices in exploratory factor analysis: Four Recommendations for Getting the Most from Your Analysis. Practical Assessment, Research \& Evaluation, 10 (7). Recuperado el 19 de Agosto de 2017 de http://pareonline.net/pdf/v10n7.pdf

Coulacoglou, C., \& Saklofske, D. (2017). Psychometrics and psychological assessment. Principles and applications. London: Academic Press.

Crosby, A. E., Ortega, L., \& Melanson, C. (2011). Self-directed violence surveillance: Uniform definitions and recommended data elements, 
version 1.0. Atlanta (GA), USA: Centers for Disease Control and Prevention, National Center for Injury Prevention and Control.

Desuque, D., Vargas-Rubilar, J., \& Lemos, V. (2011). Análisis psicométrico del cuestionario de creencias actitudinales sobre el comportamiento suicida en población adolescente en entre ríos, Argentina. Liberabit, 17(2), 187-198.

Ellis, T. E. (2008). Cognición y Suicidio. Teoría, investigación y terapia. México: Manual Moderno.

Federación de Psicólogos de la República Argentina. (2013). Código de Ética de la Fe.P.R.A. Recuperado el día 17 de marzo de 2015 de http://fepra.org.ar/docs/acerca_fepra/codigo_d e_etica_nacional_2013.pdf

Fernández-Liporace, M., \& Casullo, M. M. (2006).Validación factorial de una escala para evaluar riesgo suicida. Revista Iberoamericana de Diagnóstico y Evaluación - e Avaliação Psicológica, 1(21), 9- 22.

Galarza, A. L., Martínez-Festorazzi, V. S., Castañeiras, C. E., \& Posada, M. C. (2014). Riesgo suicida y resiliencia en un estudio con adolescentes argentinos. Acta Psiquiátrica y Psicológica de América Latina, 60(2), 98-107.

Goldston, D. B. (2000). Suicide assessment with children and adolescents. [NIMH Tech. Rep. No. 263-MD-909995]. Recuperado de http://www.suicidology.org/c/document_librar y/get_file?folderId=235\&name=DLFE141.pdf

Green, S., Thompson, M., Levy, R., \& Lo, W. (2015). Type I and Type II error rates and overall accuracy of the revised parallel analysis method for determining the number of factors. Educational and Psychological Assessment, 75(3), 428-457.

Gvion, Y., \& Apter, A. (2012). Suicide and suicidal behavior. Public Health Reviews, 34(2).

Hair, J. F., Anderson, R. E., Tatham, R. L., \& Black, W. C. (1999). Análisis multivariante. Madrid: Prentice-Hall. 5ta. Ed.

Hair, J., Hut, G., Ringle, C., \& Sarstedt, M. (2016). A primer an partial least squares structural equation modeling (PLS-SEM). New York: SAGE.
Hamza, C. A., Stewart, S. L., \& Willoughby, T. (2012). Examining the link between nonsuicidal self-injury and suicidal behavior: A review of literature and an integrated model. Clinical Psychology Review, 32, 482495.

Hancock, G., \& Mueller, R. (2013). Structural equation modeling. Madison: IAP.

Herrero, J. (2010). El análisis factorial confirmatorio en el estudio de la estructura y estabilidad de los instrumentos de evaluación: Un ejemplo con el Cuestionario de Autoestima CA-14. Psychosocial Intervention, 19(3), 289-300.

Holgado-Tello, F., Chacón-Moscoso, S., BarberoGarcía, I., \& Vila-Abad, E. (2010). Polychoric versus Pearson correlations in exploratory and confirmatory factor analysis of ordinal variables. Quality \& Quantity, 44, 153-166.

Hooper, D., Coughlan, J., \& Mullen, M. (2008). Structural equation modelling: Guidelines for determining model fit. Electronic Journal of Business Research Methods, 6(1), 53-60.

Horn, J. L. (1965). A rationale and test for the number of factors in factor analysis. Psychometrika, 30(2), 179-185.

Horton, S. E., Hughes, J. L., King, J. D., Kennard, B. D., Westers, N. J., Mayes, T. L., \& Stewart, S. M. (2016). Preliminary examination of the interpersonal psychological theory of suicide in an adolescent clinical sample. Journal of Abnormal Child Psychology, 44(6), 11331144. doi:10.1007/s10802-015-0109-5

Hoyle, R. (2012). Handbook of structural equation modeling. New York: Guilford Press.

Huanca-Morales, J. S. (2017) Comportamientos de riesgo suicida en adolescentes escolarizados de las instituciones educativas "Comercial 45" Puno y Gran Unidad Escolar "José Antonio Encinas" Juliaca. Tesis de grado. Facultad de Enfermería. Universidad Nacional del Altiplano: Perú.

International Test Commission. (2010). International Test Commission Guidelines for Translating and Adapting Tests. Recuperado de http://www.intestcom.org/upload/sitefiles/40.pdf 
Joiner, T. E. (2005). Why people die by suicide. Cambridge, MA: Harvard University Press.

Kapur, N., Cooper, J., O’Connor, R., \& Hawton, K. (2013). Non-suicidal self-injury v. attempted suicide: New diagnosis or false dichotomy? The British Journal of Psychiatry, 202, 326-328. doi:10.1192/bjp.bp.112.116111

King, J. D., \& Kowalchuk, B. (1994). ISO30.Adolescent Inventory of Suicide Orientation-30. Minneapolis, EE.UU.: National Computer Systems.

Kline, R. (2011). Principles and practice of structural equation modeling. New York: Guilford Press.

Li, C. (2016). Confirmatory factor analysis with ordinal data: Comparing robust maximum likelihood and diagonally weighted least squares. Behavior Research Methods, 48(3), 936-949.

Martínez Arias, R. (2014). Psicometría: Teoría de los test psicológicos y educativos. Madrid: Síntesis.

Ministerio de Salud de la Nación. (2017). Estadísticas vitales. Información básica año 2016 (ISSN 1668-9054. Serie 5-N ${ }^{\circ}$ 58). Recuperado el día 1 de abril de 2018 de http://www.deis.gov.ar/Publicaciones/Archivo s/Serie5Nro58.pdf

Montero, E., \& León, O. G. (2007). A guide for naming research studies in Psychology. International Journal of Clinical and Health Psychology, 7(3), 847-862.

Muñiz, J., Elousa, P., \& Hambleton, R. (2013). Directrices para la traducción y adaptación de los tests: segunda edición. Psicothema, 25(2), 151-157.

O'Connor, R. C., \& Nock, (2014). The psychology of suicide behavior. Lancet Psychiatry, 1, 73-85. doi:10.1016/S2215-0366(14)70222-6.

O'Connor, R. C., Fraser, L., White, M. C., MacHale, S., \& Masterton, G. (2008). A comparison of specific positive future expectancies and global hopelessness as predictors of suicidal ideation in a prospective study of repeat self-harmers. Journal of Affective Disorders, 110, 207-214.

Opperman, K., Czyz, E. K., Gipson, P. I., \& King, C. (2015). Connectedness and perceived burdensomeness among adolescents at elevated suicide risk: An examination of the interpersonal theory of suicidal behavior. archives of Suicide Research, 19(3), 385-400, doi:10.1080/13811118.2014.957451

Orbach, I., Milstein, I., Har-Even, D., Apter, A., Tiano, S., \& Elizur, A. (1991). Multi-Attitude Suicide Tendency Scale for Adolescents. Journal of Psychological Assessment, 3(3), 398-404.

Organización Mundial de la Salud (2018). Public health action for the prevention of suicide. Recuperado el día 23 de febrero de 2018 de http://apps.who.int/iris/bitstream/10665/75166 /1/97892 41503570_eng.pdf

Osman, A., Gutierrez, P. M., Barrios, F. X., Bagge, C. L., Kopper, B. A., \& Linden. A. (2005). The inventory of Suicide Orientaion30. Further validation with adolescent psychiatric inpatients. Journal of Clinical Psychology, 61, 481-497.

Paniagua-Suárez, R. E., González-Posada, C. M., \& Rueda-Ramírez, S. M. (2014). Orientación al suicidio en adolescentes en una zona de Medellín, Colombia. Revista Facultad Nacional de Salud Pública, 32(3), 314-321.

Paniagua-Suárez, R. E., González-Posada, C. M., \& Rueda-Ramírez, S. M. (2016). Validation of the Spanish Version of the Inventory of Suicide Orientation - ISO 30 in Adolescent Students of Educational Institutions in Medellin - Colombia. World Journal of Education, 6(4), 22-29.

doi:10.5430/wje.v6n4p22.

Peña-Castro, G., Cordero-Tapia, S., \& TapiaCuzco, P. (2009). Estudio del riesgo de suicidio en estudiantes de las escuelas de Medicina y Psicología de la Universidad del Azuay. Tesis de grado. Universidad del Azuay: Ecuador.

Senado y Cámara de Diputados de la Nación (2015). Ley Nacional de Prevención del Suicidio No27.130. Recuperado de http://www.diputados.gov.ar/secparl/dgral_inf o_parlam entaria/dip/legislaciónNacional.html

Yong, A., \& Pearce, S. (2013). A beginner's guide to factor analysis: Focusing on exploratory factor analysis. Tutorials in Quantitative Methods for Psychology, 9(2),1 79-94. 